\title{
Gender Differences in Smoking Behaviors are Narrowing but Health Impact is Wider in People Living with HIV
}

\author{
Maria Jose Miguez, Diego Bueno, Clery Quiros, Cassandra A. Stanton, Jose Castro, \\ and Christopher W. Kahler
}

\section{ABSTRACT}

The widespread use of tobacco among people living with HIV (PLWH) has a significant impact on morbidity and mortality. However, gender differences in cigarette smoking and its consequences among PLWH have not been documented. For these analyses, we included 470 PLWH smokers (255 males and 215 females) actively followed in a randomized clinical trial. A comprehensive smoking and medical profile were obtained along with FEV 1 to assess if lung function and subsequent reduction differed by gender. Almost half of the participants were females $(47 \%)$ and were slightly younger than their male counterparts $(49 \pm 9$ vs. $52 \pm 9$ years, $p=0.009)$. Smoking onset was similar $(17 \pm 7$ vs. $18 \pm 7$ years of age, $p=0.1)$, as well demonstrated preferences for mentholated cigarettes (women $89 \%$ vs. men $85 \%$ ). Based on pulmonary function testing, females had a significantly lower percent predicted forced expiratory volume in one second $(F E V 1=1.38)$ versus 1.9 in the male counterparts. Furthermore, women had a greater reduction in FEV1 over time. Among a clinical sample of PLWH smokers, we demonstrated significantly greater manifestations of lung damage in females compared to male smokers. Collectively, these findings further justify the need for more studies to closely monitor and tailor interventions for women.

Keywords: gender, HIV, lung function, smoking.

\section{INTRODUCTION}

Despite significant reductions in smoking in the general population, people living with HIV (PLWH) have rates ranging from 40.5 to $84 \%$. As a result, tobacco has emerged as a leading killer of people living with HIV. Examining smoking behavior in women living with HIV has become increasingly relevant as their prevalence continues to rise. They also seem to have more difficulties quitting smoking.

Beyond smoking behaviors, it is important to monitor health changes because smoking has been associated with several chronic medical conditions, particularly cardiovascular and respiratory diseases. Additional studies are also needed because whether or not there are differential gender risks remains controversial. Some studies have documented an accelerated decline in lung function among women [1]-[3], whereas other studies found quite the opposite [4]. Given the lack of consensus, a better understanding is essential to the future management of
Submitted : November 16, 2020

Published : February 19, 2021

ISSN: 2593-8339

DOI: 10.24018 / ejmed.2021.3.1.574

M. J. Miguez*

Florida International University, Miami, FL, USA.

(e-mail: mjmiguez@ fiu.edu)

D. Bueno

Florida International University, Miami,

FL, USA.

(e-mail: dabueno@fiu.edu)

C. Quiros

Florida International University, Miami,

FL, USA.

(e-mail: quirosc ${ }^{@}$ fiu.edu)

C. Stanton

Georgetown University, Washington,

DC, USA.

(e-mail: cas337@georgetown.edu)

J. Castro

University of Miami, Miami, FL, USA.

(e-mail: JCastro2@med.miami.edu)

C. Kahler

Brown University School of Public Health, Providence, RI, USA. (e-mail:

christopher_kahler@brown.edu)

*Corresponding Author smokers.

Understanding gender differences in smoking is important for several reasons. First, notwithstanding a small number of studies, little is known about the epidemiology of gender differences in smoking among people living with HIV [5], [6]. Smoking is the lead cause of morbidity and mortality and if gender differences exist this would have important implications for tobacco control interventions [7], [8]. For example, we expected gender differences to have been narrowed (i.e., women would report smoking the same or more than men). Furthermore, as a result of smoking mentholated cigarettes, we expect higher rates of obesity, which will further impact outcomes.

Our ongoing smoking cessation clinical trial of PLWH in Miami represents an ideal population in which to explore these gender differences because the enrollment of male and females are very similar and our cohort of HIV+ smokers underwent a thorough evaluation of smoking parameters. 


\section{METHODS}

\section{A. Study Participants}

The PATCH Study is an ongoing randomized clinical trial study of HIV+ smokers in Miami, Florida that began in May 2016. Adults were eligible once HIV, smoking status, and willingness to quit smoking were confirmed. For safety reasons, subjects were excluded if they had any contraindication to nicotine patches or gums, were involved in any other smoking and/or drug cessation or weight control programs, or had comorbid conditions that limited their safe participation, such as presence of psychotic or disabling psychiatric disorders. Drug and alcohol use were limited to those that used it recreationally, and its potential confounder effect was examined in the analyses.

Participants were recruited via three strategies: (1) by contacting participants while visiting the health care clinics, (2) using flyers, and (3) by word of mouth. Interested smokers phoned a central research office where they completed a telephone screening with a research team member to determine eligibility. Participants then underwent an inperson pre-screening, and those who qualified and completed enrollment procedures (signing consent and HIPAA forms) completed baseline procedures the same day. Written study materials, informed consent forms, and the study protocol were approved by Western IRB (WIRB). All procedures occurred at the University of Miami Clinical Translational Research Site.

To date, 470 subjects have completed the baseline evaluation, which focuses on profiling the sociodemographic, medical, and smoking habits of the individual. After completing the smoking surveys and medical assessments, blood was drawn. Final Stage.

When you submit your final version, after your paper has been accepted, prepare it in two-column format, including figures and tables.

\section{B. Smoking Surveys}

Subjects completed several standardized surveys to profile smoking history. Smoking history included core questions related to tobacco prevalence and consumption, cessation, second-hand smoke, media and advertising exposure, and economics. In the first section, participants were asked regarding the age of initiation, number of years smoking, type of tobacco used (i.e., cigarettes, length, mentholated, use of filters, cigars, pipes), and average number of cigarettes smoked per day at the beginning and thereafter (10 years interval). These data, along with age of initiation and total number of years of smoking, enabled estimation of cumulative exposure. Surveys also assessed readiness to quit, motivations, number of prior attempts to quit, their motivation and the method used.

Type of Cigarettes: Core questions regarding type of cigarettes used included preference for full, light, or ultralight cigarettes, mentholated or non-mentholated (yes/no), and the participants' preferred brands.

Finally, participants complete the Quality of Life Smoking Questionnaire where participants rate their overall health and the physical limitations for physical activities.

To measure nicotine dependence, we selected the Fagerström Test for Nicotine Dependence (FTND) because it is reliable and for its availability in English and Spanish [9].
Scores from the FTND ranged from 0 to 10 and were classified as low nicotine dependence (score 0-3), moderate nicotine dependence (score 4-6), and high dependence (score 7-10).

\section{Main Outcome: Tobacco Exposure Instruments and Tests}

Participants were advised to avoid exercise for 24 hours before their initial assessment. Before taking measures, participants were asked to relax and loosen their tight clothing. All data collectors were trained in the use of spirometers, which were newly purchased at the start of the study. The $\mathrm{CO}_{2}$ and the lung age tests were demonstrated to the participants before obtaining measures, and results were recorded once it was confirmed that there were no errors in execution.

The subjects were instructed to stand and take a deep inspiration, to hold their breath for 15 seconds, and to forcefully expire into the mouthpiece of the Smokerlyzer. This process was repeated using the Bedfont Scientific LungLife Spirometer measurement.

The Smokerlyzer ${ }^{\circledR}$, non-invasively measures the amount of $\mathrm{CO} 2$ on a smoker's breath. The machine also automatically calculates the best FEV1 from multiple blows, and then with the input of sex, height, and FEV6 measures, the LungLife Spirometer calculates lung age based on the sex-specific ECSC equations.

Based on participant's height, weight, and race, the equipment converts the FEV value to a percentage of normal. These values are also considered a good marker for the degree of obstruction and were used to classify participants based on the following.

- FEV1 greater $80 \%$ of predicted= normal-GOLD 1 .

- FEV1 $50 \%$ to $79 \%$ of predicted = Mild obstruction GOLD 2.

- FEV1 $30 \%$ to $49 \%$ of predicted = Moderate obstructionGOLD 3.

- FEV1 less than $30 \%$ of predicted = Severe obstructionGOLD 4.

The equipment is calibrated regularly. In addition, during testing, if the blow is not good, there is a slow start of the test, or cough is detected, the equipment provides an alert to repeat the test. The manufacturer reports the accuracy of the Vitalograph to be $< \pm 3 \%$. It has a Maximum Recorded Volume of 9.99 and a maximum recorded flow rate of 960 liters/min.

Finally, cotinine levels were measured using an enzyme immunoassay (OraSure Technologies, Bethlehem, PA), which is calibrated across a range from 2 to $50 \mathrm{ng} / \mathrm{mlL}$.

\section{Nutritional and Anthropometric Measures}

Height and weight were measured directly to calculate Body Mass Index (BMI) and to determine general obesity (BMI= weight in kilograms divided by the square of height in meters). Waist circumference was also obtained as an additional measure for central obesity, which has been reported as a predictor of pulmonary function.

\section{E. Other Covariates}

Sociodemographic information (sex, ethnicity, education, income, marital status, and employment) was measured by 
means of self-report at each examination. In addition, the following HIV associated variables were assessed: time since diagnosis, time receiving antiretroviral therapy, HIV-specific clinical history, CD4s, and viral load (AMPLICOR HIV-1 monitor test, Roche Diagnostics, Branchburg, NJ).

\section{F. Statistical Analysis}

SPSS version 23.0 (SPSS, IBM Inc., Armonk, NY) was used for analysis. Descriptive analyses were conducted to present demographics and clinical data. We compared demographic characteristics, smoking history, and clinical measures between males and females using ANOVA, chisquare test, or the Wilcoxon rank sum test when analyzing categorical versus continuous variables.

\section{RESULTS}

\section{A. Demographic Characteristics}

The male $(n=211)$ to female $(n=182)$ ratio was nearly one to one (1.16). As a reflection of current trends of the HIV epidemic, the mean age was above 50 years in both groups; however, the extremes of the age distribution were 23 and 69 years old. As depicted in Table I, women were marginally younger and had higher body mass index. Males and females did not differ in any other sociodemographic parameters (income, education).

At baseline, all participants were receiving antiretroviral treatment, yet they differed in some clinical parameters. Although gender was not associated with self-reported adherence, or virologic control of the disease, women had higher CD4 cell counts. Of relevance, body mass index was significantly higher among females, who were more likely to have BMI above $30 \mathrm{~kg} / \mathrm{m}^{2}$.

\begin{tabular}{cccc}
\multicolumn{4}{c}{ TABLE I: DEMOGRAPHIC CHARACTERISTICS OF STUDY PARTICIPANTS } \\
\hline Variable & Men & Women & P-value \\
\hline Age & $52.2 \pm 9.1$ & $49.6 \pm 8.7$ & 0.05 \\
African American & $80 \%$ & $84 \%$ & \\
Hispanic & $11 \%$ & $6 \%$ & \\
Caucasian & $7 \%$ & $10 \%$ & $<0.03$ \\
Other & $2 \%$ & $0 \%$ & \\
& $75 \%$ & $79 \%$ & \\
Income & $22 \%$ & $18 \%$ & 0.6 \\
& $3 \%$ & $3 \%$ & 0.78 \\
Education & $5.5 \pm 1.9$ & $5.6 \pm 2.1$ & 0.00 \\
BMI & $26.6 \pm 5.6$ & $30.5 \pm 8.2$ & 0.00 \\
CD4 & $493 \pm 320$ & $651 \pm 456$ & 0.2 \\
Viral Load & $73 \%$ & $76 \%$ & \\
Undetectable & & &
\end{tabular}

\section{B. Gender Differences in Smoking Behaviors}

Both males and females had been daily smokers. Table II shows men smoked on average 14.7 cigarettes per day while women smoked 13.7, indicating that differences by gender have narrowed. Both groups started smoking at early ages, males at 18 years of age (SD 6.6) and women slightly before $(17 \pm 7, p=0.2)$. Yet, when they started, males smoked slightly more, but not too much more than their female counterparts $(8 \pm 0.5$ vs. $6 \pm 0.4, p=0.03)$. They both had been smokers for approximately 5 decades (52 vs. 49). Data indicated that our smoking sample in South Florida overwhelmingly preferred mentholated brands $(86 \%$ versus $\approx 25 \%$ US general population). The rate of menthol use was $88 \%$ among females versus $85 \%$ among males.

The gender based analyses of reasons to smoke revealed that women were more likely than men to report smoking for affect regulation, specifically to control their stress levels $(\mathrm{OR}=2.895 \% \mathrm{CI} 1.37-5.7 ; \mathrm{p}<0.001)$. Women were also more likely to smoke as a means to control their weight $(\mathrm{OR}=1.57$ 95\% CI 1-2.5, $\mathrm{p}=0.04)$.

TABLE II: CigARETTE USE AND LUNG PARAMETERS BY GENDER

\begin{tabular}{cccc}
\hline Status & Men & Women & P-value \\
\hline \# Cigarettes per day & $14.7 \pm 10.0$ & $13.7 \pm 9.5$ & 0.3 \\
Fargerstrom & $5.1 \pm 2.1$ & $5.6 \pm 1.7$ & 0.01 \\
Quit Ladder & $6.9 \pm 0.79$ & $6.8 \pm 0.9$ & 0.2 \\
Years Smoking & $30.0 \pm 10.5$ & $31.0 \pm 11.2$ & 0.5 \\
Chronological Age & $52.3 \pm 9.1$ & $49.6 \pm 9.1$ & 0.005 \\
FEV1 & $2.1 \pm 0.7$ & $1.5 \pm 0.5$ & 0.003 \\
\hline
\end{tabular}

\section{Gender Differences on Cotinine}

Cotinine levels were significantly correlated with the nicotine dependence score $(\mathrm{r} 2=.21, \mathrm{p}<.0001)$, with the body mass index $(\mathrm{r} 2=-0.118, \mathrm{p}=.002)$, and the use of mentholated cigarettes $(\mathrm{r} 2=0.119, \mathrm{p}=.002)$. Yet, it was not significantly related to number of cigarettes smoked per day ( $\mathrm{r} 2=.07$, $\mathrm{p}=.130$ ). Cotinine levels were slightly higher among females but did not reach statistical significance (192.2 \pm 131 vs. $185.0 \pm 116 \mathrm{ng} / \mathrm{ml} \mathrm{p}=.57)$. This may be related to the fact that obese individuals had lower cotinine levels than their nonobese counterparts, $(163.1 \pm 113$ vs. $201.7 \pm 126 \mathrm{ng} / \mathrm{ml}$ $\mathrm{p}=.003)$.

\section{Dependence}

The scores from the FTND in the total group were in the moderate levels of dependence; nonetheless $24 \%$ scored at the high dependence levels (score 7-10). Despite exhibiting similar cotinine levels, females scored significantly higher in the FTND. Among the group, $60 \%$ of the males had a medium or strong nicotine dependence, while $78 \%$ of the female smokers scored above 5 on the FTND. In other words, females were twice more likely to have medium to high levels of dependency than their male counterparts $(\mathrm{OR}=2.3,95 \%$ CI $1.2-4.3 \mathrm{p}=0.001$ ).

\section{E. Quit Attempts}

Despite the observed differences in nicotine dependence, male and females were equally motivated to quit smoking. Scores in the quit ladder were similar (males $=6.87 \pm 0.7$ vs. $6.97 \pm 0.8 ; \mathrm{p}=0.23)$. Women had in average similar numbers of lifetime quit attempts to men (3.7 \pm 2.1 vs $3.4 \pm 1.8)$, yet a higher percent of men reported more than five attempts $(34.5$ vs 25.7). Based on pulmonary function testing, females had a lower percent predicted forced expiratory volume in one second (FEV1). The average FEV1was 1.48 in the female participants versus 1.9 in the male counterparts, and the differences remained the same after controlling for the number of cigarettes smoked. Also, female subjects had lower forced expiratory volume in $1 \mathrm{~s}$ (FEV1) \% predicted (48.7\% vs $55.8 \%, \mathrm{p}=0.001)$.

Table III demonstrates clinical pulmonary findings according to participant's gender. Despite similar number of cigarettes per day and equal number of years smoking, females reported more physical restrictions compared to male smokers living with HIV. Women showed more severe limitations climbing stairs and walking long distances than men. 
TABLE III: CLINICAL SYMPTOMS BY GENDER

\begin{tabular}{cccccccc}
\hline & \multicolumn{3}{c}{ Male Limited } & \multicolumn{3}{c}{ Female } \\
\cline { 2 - 6 } $\begin{array}{c}\text { Grading } \\
\text { Limitations }\end{array}$ & $\begin{array}{c}\text { A lot } \\
\%\end{array}$ & $\begin{array}{c}\text { A } \\
\text { little } \\
\%\end{array}$ & $\begin{array}{c}\text { Not } \\
\text { at all } \\
\%\end{array}$ & $\begin{array}{c}\text { A lot } \\
\%\end{array}$ & $\begin{array}{c}\text { A } \\
\text { little } \\
\%\end{array}$ & $\begin{array}{c}\text { Not } \\
\text { at all } \\
\%\end{array}$ & $\begin{array}{c}\text { P- } \\
\text { value }\end{array}$ \\
\hline $\begin{array}{c}\text { Vigorous } \\
\text { activities } \\
\text { Lifting }\end{array}$ & 31 & 32 & 37 & 38 & 33 & 29 & 0.05 \\
$\begin{array}{c}\text { groceries } \\
\text { Climbing } \\
\text { Stairs }\end{array}$ & 12 & 39 & 49 & 17 & 41 & 42 & 0.06 \\
$\begin{array}{c}\text { Walking } \\
\text { more than } 1\end{array}$ & 27 & 35 & 38 & 38 & 33 & 29 & 0.01 \\
$\begin{array}{c}\text { mile } \\
\text { Walking } \\
\text { several } \\
\text { blocks }\end{array}$ & 17 & 31 & 52 & 25 & 34 & 42 & 0.03 \\
Bathing & 6 & 8 & 86 & 7 & 11 & 81 & 0.35 \\
\hline
\end{tabular}

\section{DISCUSSION}

Our study demonstrated dramatically high rates of smoking among PLWH, particularly among females. These findings have important clinical implications: First, because one can expect that women (particularly as they age) will be more susceptible to tobacco's deleterious health effects. Secondly, increases in nicotine have been associated with vulnerability to nicotine addiction and highlights the need for additional attention during times known for hormonal alterations (i.e., menopause, pregnancy and following hysterectomy). Thirdly, these results also have some treatment connotations as they suggest the need for dose adjustments during pharmacological interventions. Evidencebased tobacco control policies should be implemented to stop the rise in women's smoking rates worldwide. Following the WHO Framework Convention on Tobacco Control (FCTC), a greater emphasis should be placed on policies aimed to prevent the tobacco industry from targeting women, including bans on all forms of tobacco advertising and promotion [10].

Few small-scale studies have assessed gender differences on nicotine and cotinine clearance, with varying results [11]. Some have reported no differences, while others have demonstrated shorter cotinine half-life in females, compared to males. Prather and colleagues also found a quicker elimination of nicotine in females [12]. These investigations are in line with our findings of gender differences on tobacco biomarkers, even when BMI was taken into consideration. However, it contrasts with Benowitz, who postulates that gender differences in nicotine levels are mostly related to body composition [13]. The opposite occurred in PLWH; HIV-infected females exhibited the highest cotinine levels. A possible explanation is that beyond drug interference, hormonal disarrangements, which are frequent among HIVinfected females, could be further slowing the nicotine metabolism [11], [14]. Supporting this postulate is our distressing finding that menopausal females, and those that have had hysterectomies, displayed the highest cotinine levels. However, supporting laboratory data or larger sized studies are needed to confirm or reject our hypothesis. This finding, though preliminary, may have clinical implications for post-menopausal females who attempt to quit smoking.

In addition, it is reasonable to hypothesize that in these female's morbidity and mortality from tobacco-related disease would significantly increase. Thus, it is of utmost importance for the US government to take a precautionary approach and re-assesses the idea of banning menthol as an ingredient in tobacco to reduce smoking uptake among PLWH, particularly among females.

Although the study sample was limited to the clinical settings of South Florida, and current analyses were based on baseline information, our study benefitted from its mid-sized sample. The large number of females involved in the study, the use of non-mentholated cigarettes, and the direct measuring of cotinine (rather than relying on self-reported data) makes this study notable.

\section{CONCLUSION}

The dangers of smoking are well established, but certainly our analyses suggest that use of mentholated cigarettes can be adding additional risks above and beyond those of nonmenthol flavored cigarettes. Given our results, a public health mandate to ban menthols would benefit all, but most especially those living with HIV. The dangers of tobacco products and the excessive rates of menthol use are troubling. The aging of our HIV infected population further justifies the need for more studies to closely monitor the public health threat of mentholated cigarette smoking. Additionally, hormones significantly affect nicotine and cotinine metabolism, another indication of the need for more genderbased studies among PLWH.

\section{ACKNOWLEDGMENT}

We are indebted to all participants in this study for their cooperation. Also, we thank the University of Miami Clinical Translational Research Site for their support during the survey. The Research Project was fully sponsored by the Florida Department of Health with grant number (6JK04).

\section{REFERENCES}

[1] D.M. MacDonald, A.C Melzer, G. Collins, A. Avihingsanon, K Crothers, and N.E. Ingraham, "INSIGHT START Pulmonary Substudy Group. Smoking and accelerated lung function decline in HIV-positive individuals: a secondary analysis of the START pulmonary substudy". J Acquir Immune Defic Syndr, 79:e85-e92. 2018.

[2] S. H. Downs, O. Brändli, J. P. Zellweger, et al. "Accelerated decline in lung function in smoking women with airway obstruction: SAPALDIA 2 cohort study." Respiratory research, 6(1), 45. 2005.

[3] M. Tommola, P. Ilmarinen, and L.E. Tuomisto, "The effect of smoking on lung function: a clinical study of adult-onset asthma." Eur Respir J, 48: 1298-1306. 2016.

[4] W.M. Vollmer, P.L. Enright, and K.L. Pedula, "Race and gender differences in the effects of smoking on lung function." Chest, 11(7), $764-772.2000$

[5] V.E. Bryant, C.W. Kahler, K.N. Devlin, et al. "The effects of cigarette smoking on learning and memory performance among people living with HIV/AIDS.” AIDS Care. 25(10), 1308-16. 2013.

[6] H. Pines, L. Koutsky, and S. Buskin, "Cigarette smoking and mortality among HIV-infected individuals in Seattle, Washington (1996-2008)." AIDS Beh., 15(1), 243-51. 2011.

[7] Centers for Disease Control and Prevention. Tobacco Related Mortality. National Center for Chronic Disease Prevention and Health Promotion. Office on Smoking and Health. [accessed 2019 Feb 20].

[8] U.S. Department of Health and Human Services. (2004). Department of Health and Human Services, Centers for Disease Control and Prevention; Atlanta, GA: The health consequences of smoking: A report of the Surgeon General. 
[9] T.F. Heatherton, L.T. Kozlowski, R.C. Frecker, and K.O. Fagerstrom, "The fagerstrom test for nicotine dependence: A revision of the fagerstrom tolerance questionnaire." Br J Addict, 86, 1119-1127. 1991.

[10] World Health Organization. (2003). WHO Framework Convention on Tobacco Control. Geneva: World Health Organization.

[11] J. Hukkanen, I.P. Jacob, and N.L Benowitz, "Metabolism and disposition kinetics of nicotine." Pharmacol Rev, 57(1), 79-115. 2005.

[12] R.D. Prather, T.G. Tu, C.N. Rolf, et al. "Nicotine pharmacokinetics of Nicoderm ${ }^{\circledR}$ (nicotine transdermal system) in women and obese men compared with normal-sized men." J Clin Pharmacol, 33, 644-9. 1993.

[13] N.L. Benowitz, and P. Jacob, "Metabolism of nicotine to cotinine studied by a dual stable isotope method." Clin Pharmacol Ther, 56, 483-493. 1994.

[14] M.D. Fan, B.S. Maslow, N. Santoro, and E. Schoenbaum, "HIV and the menopause." Menopause Int., 14, 163-8. 2008. 